\title{
A Review on Road Extraction based on Neural and Non-Neural Networks
}

\author{
D. Subhashini \\ Dept of ECE, \\ MGIT,Hyderabad
}

\author{
Dr. V. B. S. Srilatha Indira Dutt \\ Dept of ECE \\ GITAM University, Vishakapatnam
}

\begin{abstract}
Satellite images play a vital role in our daily life and are used in various remote sensing applications, such as geography, forestry, water conservancy, marine, natural disasters etc. During satellite image processing, GIS (Geographic information system) plays a vital role with road extraction. Road is an important kind of basic geographic information. Road information extraction plays an important role in traffic management, urban planning, automatic vehicle navigation, and emergency management. With the development of remote sensing technology, the quality of high-resolution satellite images is improved and more easily obtained, which makes it possible to use remote sensing images to locate roads accurately. Therefore, it is an urgent problem to extract road information from remote sensing images In this paper we propose different methodologies in road extraction and their comparisons with respect to completeness, correctness and quality. The comparison is taken based on semi-automatic method and automatic method, Neural Network and Non neural networks methodologies.
\end{abstract}

\section{Keywords--Road Extraction, completeness, correctness and} quality.

\section{I.INTRODUCTION}

Automatic detection of road links from the satellite images is one of the demanding research topics and it is used for many applications. Geographic information system (GIS) needs an automatic road extraction process for updating their data[1]. Manual updating is costly, time-consuming process and also there is a chance of error in manual updating of road network [2][3][4]. So, automatic road feature extraction from high resolution satellite image is required in a robust manner. High resolution remote sensing image has been widely used in agriculture and forestry, water conservancy, geography, geology, surveying and mapping, geology and mineral resources, Marine, land, global change, natural disasters, environmental protection, military reconnaissance, and other fields, and plays an important role in the economic, social and national defense construction and scientific research. Road data permits GIS applications to facilitate different services like satellite navigation, route planning, health-care accessibility planning, landscaping and infrastructure management. The major problem of road extraction method is the complex structure of the images, which contain many objects, such as roads, houses, trees, vehicles, etc., with differences in shape, size and the texture.

Road extraction methods can be classified into two categories such as semi-automatic and fully automatic[5]. The road detection techniques which require human interaction are considered as semi-automatic[6], and those that are not requiring human interaction are known as automatic. Though many researchers are developing algorithm for road detection, as semi-automatic[7] which is not suitable for real-time application and other works are automatic road detection system[8] All the work has its own merits and demerits.

\section{SATELLITE IMAGERY}

Satellite imagery is images of Earth or other planets collected by imaging satellites operated by governments and businesses around the world. Satellite imaging companies sell images by licensing them to governments and businesses such as Apple Maps and Google Maps.

There are four types of resolutions when discussing satellite imagery in remote sensing: spatial, spectral, temporal, radiometric and geometric. Spatial resolution is defined as the pixel size of an image representing the size of the surface area .Spectral resolution is defined by the wavelength interval size (discrete segment of the Electromagnetic Spectrum) and number of intervals that the sensor is measuring. Temporal resolution is defined by the amount of time (e.g. days) that passes between imagery collection periods for a given surface location. Radiometric resolution is defined as the ability of an imaging system to record many levels of brightness (contrast for example) and to the effective bit-depth of the sensor (number of grayscale levels) and is typically expressed as 8-bit (0-255), 11-bit (0-2047), 12-bit (0$4095)$ or 16 -bit $(0-65,535)$. Geometric resolution refers to the satellite sensor's ability to effectively image a portion of the Earth's surface in a single pixel and is typically expressed in terms of Ground sample distance, or GSD. The resolution of satellite images varies depending on the instrument used and the altitude of the satellite's orbit. Satellite imagery is sometimes supplemented with aerial photography, which has higher resolution, but is more expensive per square meter.

In these bands of images, panchromatic band is a single band, the remote sensing image of panchromatic band which has high spatial resolution displays as a grayscale image. Multiband remote sensing image can obtain the color information of ground object with low spatial resolution. 


\section{NON-CONVOLUTIONAL NEURAL NETWORK METHOD}

A.INPUT IMAGE/IMAGE ACQUISITION

Input image can be considered as satellite image, aerial image and lidar data. Compared to aerial and satellite images, airborne lidar data are less affected by obstruction and shadow, hence provide a great potential for road extraction. Common strategies for airborne lidar-based road extraction include segmentation and clustering. At first, lidar points and aerial images have to be fused on the basis of their coordinates, and the fused lidar points with red/green/blue (RGB) colour components can be partitioned into various pseudo-scanlines which are defined as buffers along the scan directions. Then an edgeclustering algorithm is proposed in order to segregate road areas from other objects. It is our aim to identify elongated road segments from the segregated lidar points by applying a set of constraints to intensity, height, elevation difference, band-ratio and length.

\section{B. WORK FLOW}

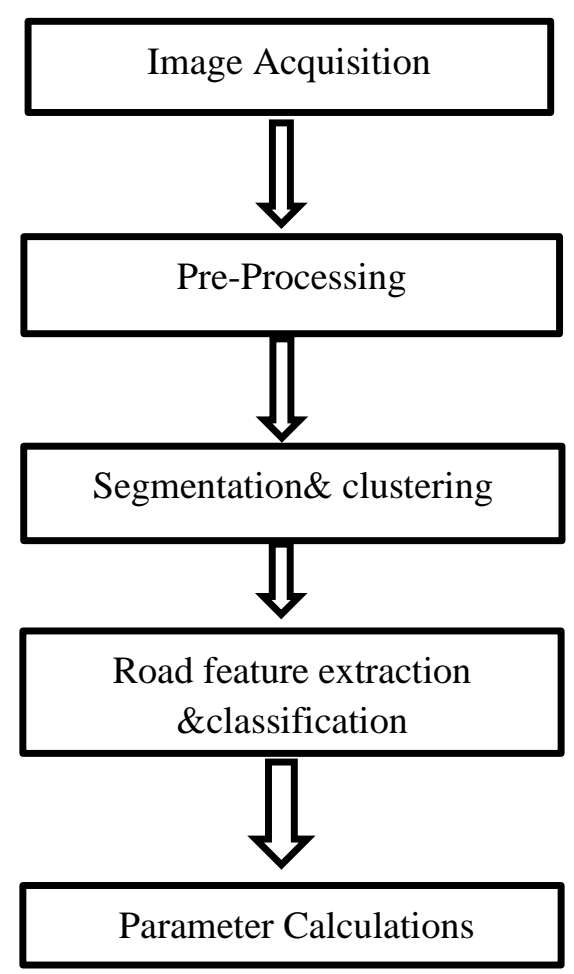

\section{PRE-PROCESSING}

The data preparation stage consists of data fusion of airborne lidar points and aerial images, and partitioning of the fused lidar points into pseudo-scanlines. To co-register and fuse lidar points and images, the corresponding row and column numbers in the images for each lidar point are calculated according to the coordinates, and the RGB values of the corresponding cell are assigned to the lidar point. The required parameter in the data fusion process is the image resolution, which is known in advance. Once the data fusion is finished, the fused lidar points have to be reorganized for the purposes of efficient data processing. Lidar data typically contains a huge number of points. Hence processing lidar data without proper data management can be both overly time-consuming and complicated.

\section{REVIEW CONCEPTS ON NON- CONVOLUTIONAL NEURAL NETWORK:}

Preprocessed satellite images /airbone /lidar images are segmented with different types of techniques. The techniques are, Thresholding-Otsu's algorithm, Fuzzy algorithm, Edge detection USM filtering, Gaussian USM filtering...etc .Depending on the obtained outputs clustering is performed as K-mean,C-mean ,Fuzzy etc.

Connected component-based technique for automatic extraction of road centerline in high resolution satellite images is presented by Chinnathevar Sujatha[9] .This work consists of Adaptive global thresholding, connected component analysis ,Morphological Closing , Opening and thinning operations.In this method the author proposed Adaptive global thresholding for segmentation, Morphological Closing operation for close the holes inside the road, Morphological Opening removal of unwanted portion and Morphological thinning operations for Road center line extraction.

A framework of road extraction from airborne lidar data and aerial imagery presented by Li Liu \& Samsung Lim[10]. The author proposed the technique based on lidar data with initial extraction by Edge Clustering, then KNN clustering, interpolation, curve fitting and refinement for 3D road points.

The Rapid Method for Road Extraction from High-Resolution Satellite Images Based on USM Algorithm presented by Liu $\mathrm{Xu}[11]$. In this paper author proposed the technique with Image preprocessing by USM algorithm, low level processing, medium level processing, and high level processing then identification of roads

Investigation of SVM and Level Set Interactive Methods for Road Extraction from Google Earth Images presented by Abolfazl Abdollah[12].In this paper author proposed the technique for conversion of RGB to HIS images with thresholding the HUE channel using OTSU's algorithm, use of HUE channel for test set of SVM (support vector Machine).

Road Network Extraction from High-Resolution Remote Sensing Image Using Homogenous Property and Shape Feature presented by Runsheng Li1[13]. In this paper author proposed method achieved the detection of potential road center points using mean shift based on homogenous property and ribbon-like shape of road. The class of detected road center points could be identified by the combination of Gabor filtering and tensor representation as it provides a good tool for the capture and analysis of high frequency information.

A Method for Automatic Road Extraction of High Resolution SAR Imagery presented by M. Saati[14]. proposed the method for multiple feature extraction of road, detection of road areas with vectorization, road extraction by Fuzzy algorithm and connection of seed points by Snake model.

Road Network Extraction using Edge Detection and Spatial Voting by Beril[15] proposed the technique as smoothing of image by bilateral filtering, Edge extraction, generation of Voting matrix and tracking operation. 
Towards Knowledge-Based Extraction of Roads from 1m-resolution Satellite Images by Hae Yeoun Lee[16], proposed this Gaussian blurring in different levels, Gradient operation and watershed operation followed by Region merging.

Extraction of Roads from High-Resolution Satellite Images with the Discrete Wavelet Transform by Tamer M. Talal [17]proposed this method using DWT for decomposition, morphological operations for length and direction identification. For this operation they had taken images from Quick Bird with $0.61 \mathrm{~m}$ resolution, IKONOS with $1 \mathrm{~m}$ resolution, and SPOT-5 with $2.5 \mathrm{~m}$ resolution.

Extraction of road using soft computing techniques by Mishmala Sushith[18] proposed B-snake with fuzzy $\mathrm{C}$-means (FCM) clustering for road lanes extraction. B-snake lane model is provided by introducing Canny/Hough estimation of vanishing points and external force field for lane detection is constructed by using gradient vector flow. Medial-axis-transform-based hypothesis verification is used to automatically complete the road network extraction framework.

Automatic Road Extraction from High Resolution Satellite Image using Adaptive Global Thresholding and Morphological Operations by Pankaj Pratap Singh [19]proposed this with segmentation technique using adaptive global thresholding and morphological operations.

An Integrated Multistage Framework for Automatic Road Extraction from High Resolution Satellite Imagery by T. T. Mirnalinee[20] proposed Support Vector Machine to discriminate between road and non-road segments. Dominant singular Measure (DSM) for the task of detecting linear (locally) road boundaries

\section{GEOMETRIC FEATURES}

Stripe features, Ratio of length and width, radiometric features, Homogeneous Surface, Contrast of road with adjacent areas.

\section{CONVOLUTIONAL NEURAL NETWORK METHOD} A. Work flow

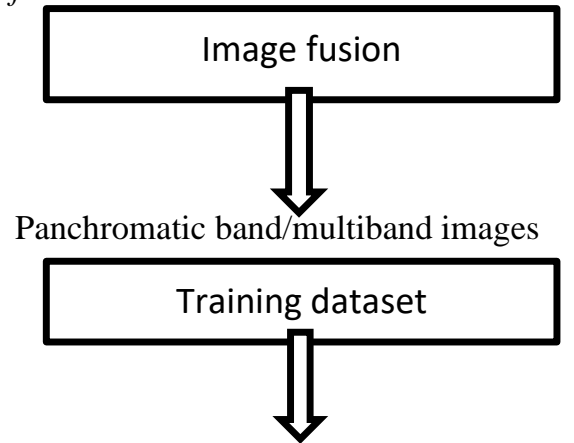

ResNet, LeNet, Google Net,Alexnet, VGG

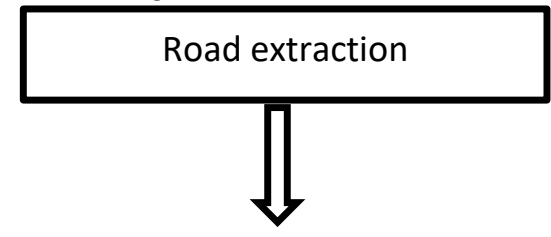

deep learning , machine learning, $\mathrm{CNN}$...

\section{Parameter calculations}

Completeness, correctness, quality

\section{B. REVIEW BASED ON CONVOLUTIONAL NEURAL NETWORK:}

Road Extraction from Multi-source Highresolution Remote Sensing Image Using Convolutional Neural Network by Yun Zhang [21] proposed the method based on deep learning network. They performed the extraction by considering training of dataset with two kinds of high resolution remote sensing data which are GaoFen-2 and World View.

Road network extraction in high-resolution SAR images based CNN features by Yue Li [22]proposed the method based CNN using high resolution SAR images. Road candidates are identified with CNN model, road segmentation with improved Radon Transform and finally road networks are identified with MRF (Markov random field).

Research on road extraction of remote sensing image based on convolutional neural network by Yuantao Jiang[23] proposed the method based on CNN using training algorithms and by using wavelets.

Road network extraction from satellite images using cnn based segmentation and tracing by Yao Wei[24] proposed based on $\mathrm{CNN}$ with segmentation and tracing i.e road line identification. For segmentation they used encoding, decoding and connection between them. Finally road center line is identified with Adaptive Starting Point Decision (ASPD) algorithm.

\section{Training Dataset}

Dataset is trained by ReNet,LetNet,GoogleNet,VGG etc. are used depending on type of satellite images/Data fusion type

\section{Road Extraction}

After performing Training Road extraction is performed by Deep Neural Network, Machine learning, CNN, AI etc.

\section{ADVANTAGES OF METHODOLOGY -I :NON NEURAL NETWORKS}

- No requirement of training data

- $\quad$ simple algorithm are sufficient

- But it requires more processing time

- less accurate outputs.

VII. ADVANTAGES OF METHODOLOGY -II : CONVOLUTIONAL NEURAL NETWORK

- Requires simple trained algorithm

- more accurate results

- it requires more training dataset

\section{CALCULATIONS AND PERFORMANCE} MEASURES

- $\quad$ Completeness $=\mathrm{TP} /(\mathrm{TP}+\mathrm{FN})$;

- $\quad$ Correctness = TP/ $(\mathrm{TP}+\mathrm{FP})$;

- Omission rate $=\mathrm{FN} /(\mathrm{TP}+\mathrm{FP})$;

- $\quad$ Commission rate $=1-\mathrm{TP} /(\mathrm{TP}+\mathrm{FP})$;

- $\quad$ Quality $=\mathrm{TP} /(\mathrm{TP}+\mathrm{FP}+\mathrm{FN})$. 
Where TP - True Positive, an element present in ground truth and extracted road network, FP - False Positive, an element present in extracted road network but not in ground truth, FN - False Negative, an element present in ground truth but not in extracted road network

TABLE1

COMPARISION BETWEEN CONVOLUTIONAL AND NON CONVOLUTIONAL NETWORKS

\begin{tabular}{|l|l|l|l|}
\hline $\begin{array}{l}\text { Type of } \\
\text { Network }\end{array}$ & $\begin{array}{l}\text { Complete } \\
\text {-ness }\end{array}$ & $\begin{array}{l}\text { Correctn- } \\
\text { ess }\end{array}$ & quality \\
\hline $\begin{array}{l}\text { convolutional } \\
\text { Neural } \\
\text { Network }\end{array}$ & Upto 90\% & Upto 96\% & $\begin{array}{l}\text { Upto } \\
87 \%\end{array}$ \\
\hline $\begin{array}{l}\text { convolutional } \\
\text { Neural } \\
\text { Network }\end{array}$ & Upto 93\% & Upto 91\% & $\begin{array}{l}\text { Upto } \\
93.1 \%\end{array}$ \\
\hline
\end{tabular}

\section{CONCLUSION}

From this survey we got to know there are different methodologies in Non-Neural Networks and Neural Networks. But in all these methodologies if we have sufficient data set it produces better and accurate results with less computing time.

\section{REFERENCES}

[1] J. Hu, A. Razdan, J. C. Femiani, M. Cui, and P. Wonka, "Road network extraction and intersection detection from aerial images by tracking road footprints," IEEE Trans. Geosci. Remote Sens., vol. 45, no. 12, pp. 4144-4157, 2007, doi: 10.1109/TGRS.2007.906107.

[2] C. Ünsalan and B. Sirmacek, "Road Network Detection Using Probabilistic.pdf," vol. 50, no. 11, pp. 4441-4453, 2012.

[3] X. Jin and C. H. Davis, "An integrated system for automatic road mapping from high-resolution multi-spectral satellite imagery by information fusion," Inf. Fusion, vol. 6, no. 4, pp. 257-273, 2005, doi: 10.1016/j.inffus.2004.06.003.

[4] Z. Huang, J. Zhang, L. Wang, and F. Xu, "A FEATURE FUSION METHOD FOR ROAD LINE EXTRACTION FROM REMOTE SENSING IMAGE 1 . School of Electronic Science and Engineering, National University of Defense Technology 2 . IIST Key Lab ., Institute of Software, Chinese Academic Science 3 . Communicatio,” pp. 52-55, 2012.

[5] X. Lin, Z. Liu, J. Zhang, and J. Shen, "Combining multiple algorithms for road network tracking from multiple source remotely sensed imagery: A practical system and performance evaluation," Sensors, vol. 9, no. 2, pp. 1237-1258, 2009, doi: $10.3390 / \mathrm{s} 90201237$.

[6] A. P. Dal Poz, R. B. Zanin, and G. M. Do Vale, "Automated extraction of road network from medium-and high-resolution images," Pattern Recognit. Image Anal., vol. 16, no. 2, pp. 239248, 2006, doi: 10.1134/S1054661806020118.

[7] Z. Miao, B. Wang, W. Shi, and H. Zhang, "A semi-automatic method for road centerline extraction from VHR images," IEEE Geosci. Remote Sens. Lett., vol. 11, no. 11, pp. 1856-1860, 2014, doi: 10.1109/LGRS.2014.2312000.

[8] Z. Miao, W. Shi, H. Zhang, and X. Wang, "Road centerline extraction from high-resolution imagery based on shape features and multivariate adaptive regression splines," IEEE Geosci.
Remote Sens. Lett, vol. 10, no. 3, pp. 583-587, 2013, doi 10.1109/LGRS.2012.2214761.

[9] C. Sujatha and D. Selvathi, "Connected component-based technique for automatic extraction of road centerline in high resolution satellite images," Eurasip J. Image Video Process., vol. 2015, no. 1, 2015, doi: 10.1186/s13640-015-0062-9.

[10] L. Liu and S. Lim, "A framework of road extraction from airborne lidar data and aerial imagery," J. Spat. Sci., vol. 61, no. 2, pp. 263-281, 2016, doi: 10.1080/14498596.2016.1147392.

[11] X. Liu, J. Tao, X. Yu, J. J. Cheng, and L. Q. Guo, "The rapid method for road extraction from high-resolution satellite images based on USM algorithm," Proc. 2012 Int. Conf. Image Anal. Signal Process. IASP 2012, pp. 96-100, 2012, doi: 10.1109/IASP.2012.6425000.

[12] "Investigation of SVM and Level Set Interactive Methods for Road Extraction from Google Earth Images," J. Indian Soc. Remote Sens., vol. 46, no. 3, pp. 423-430, 2018, doi 10.1007/s12524-017-0702-x.

[13] "Road Network Extraction from High-Resolution Remote Sensing Image Using Homogenous Property and Shape Feature," J. Indian Soc. Remote Sens., vol. 46, no. 1, pp. 51-58, 2018, doi: 10.1007/s12524-017-0678-6.

[14] "A Method for Automatic Road Extraction of High Resolution SAR Imagery," J. Indian Soc. Remote Sens., vol. 43, no. 4, pp. 697-707, 2015, doi: 10.1007/s12524-015-0454-4.

[15] B. Sirmaçek and C. Ünsalan, "Road network extraction using edge detection and spatial voting," Proc. - Int. Conf. Pattern Recognit., pp. 3113-3116, 2010, doi: 10.1109/ICPR.2010.762.

[16] H. Y. Lee, W. Park, H. K. Lee, and T. G. Kim, "Towards knowledge-based extraction of roads from $1 \mathrm{~m}$-resolution satellite images," Proc. IEEE Southwest Symp. Image Anal. Interpret., vol. 2000-Janua, pp. 171-176, 2000, doi 10.1109/IAI.2000.839594.

[17] T. M. Talal, A. El-Sayed, M. Hebaishy, M. I. Dessouky, S. A. Alshebeili, and F. E. A. El-Samie, "Extraction of roads from high-resolution satellite images with the discrete wavelet transform," Sens. Imaging, vol. 14, no. 1-2, pp. 29-55, 2013 , doi: $10.1007 / \mathrm{s} 11220-013-0078-0$.

[18] M. Sushith and S. Sophia, "Extraction of road using soft computing techniques," Soft Comput., vol. 23, no. 18, pp. 8487 8494, 2019, doi: 10.1007/s00500-019-03966-9.

[19] P. P. Singh and R. D. Garg, "Automatic Road Extraction from High Resolution Satellite Image using Adaptive Global Thresholding and Morphological Operations," J. Indian Soc. Remote Sens., vol. 41, no. 3, pp. 631-640, 2013, doi 10.1007/s12524-012-0241-4.

[20] "An Integrated Multistage Framework for Automatic Road Extraction from High Resolution Satellite Imagery," J. Indian Soc. Remote Sens., vol. 39, no. 1, pp. 1-25, 2011, doi: 10.1007/s12524-011-0063-9.

[21] Z. Wang, X. Liu, L. Liu, and J. Shi, "A method of road extraction from high resolution remote image based on Delaunay algorithms," Proc. - 2018 Int. Conf. Robot. Intell. Syst. ICRIS 2018, pp. 127-130, 2018, doi: 10.1109/ICRIS.2018.00040.

[22] Y. Li, R. Zhang, and Y. Wu, "ROAD NETWORK EXTRACTION IN HIGH-RESOLUTION SAR IMAGES BASED CNN FEATURES Department of Electronic Engineering and Information Science , USTC , Hefei , 230027 China Key Laboratory of Electromagnetic Space Information , Chinese Academy of Sciences, Hefei ," pp. 1-4.

[23] "Research on road extraction of remote sensing image based on convolutional neural network," Eurasip J. Image Video Process., vol. 2019, no. 1, 2019, doi: 10.1186/s13640-019-04267.

[24] Y. Wei, K. Zhang, and S. Ji, "Road Network Extraction from Satellite Images Using CNN Based Segmentation and Tracing," Int. Geosci. Remote Sens. Symp., pp. 3923-3926, 2019, doi 10.1109/IGARSS.2019.8898565. 